\title{
EXPERIMENTAL STUDY OF EDGE RADIATION AT HISOR
}

\author{
N. V. Smolyakov*, A. Hiraya, G. V. Rybalchenko, K. Shirasawa, T. Muneyoshi, \\ HiSOR \& Faculty of Science, Hiroshima University, Japan
}

\section{Abstract}

The measurements of spectral-angular distributions of edge radiation emitted at the fringe fields of two bending magnets are reported. These studies were conducted at HiSOR electron storage ring (Hiroshima, Japan). The radiation was observed in the near infrared, visible and near ultraviolet ranges. The recorded images, both horizontally and vertically polarized, are compared with the numerically simulated distributions of edge radiation. Good agreement is found between the measured and calculated characteristics of edge radiation.

\section{INTRODUCTION}

Electromagnetic edge radiation (ER) is generated by a relativistic charged particle when it passes through the region of a rapid change in magnetic field at the edges of the storage ring bending magnets. It was experimentally discovered in the late 1970s simultaneously and independently at Super Proton Synchrotron (SPS, CERN, Geneva) $[1,2]$ and at the electron synchrotron "Sirius" (INP, Tomsk, Russia) [3 - 5]. It is worthy of note that the physics of ER generation by relativistic protons [6 - 10] differs radically from ER generation by electrons, though some similarities in theirs properties can be found.

The first theoretical studies of ER from high-energy electrons were carried out in papers $[4,11,12]$. As the papers [11, 12] claimed, the long-wavelength ER is linearly polarized and is bound to have an intensive peaks at the angles $\pm 1 / \gamma$ about the straight section axis, where $\gamma$ is the electron's relativistic factor. More detailed theoretical analysis made in succeeding years [13 - 24] has demonstrated a great potential of ER to be used as a source of radiation in infrared - ultraviolet spectral range. However, up to now only a few experimental studies of ER have been reported [3-5, 25-28].

In this paper, results of the experimental investigation of ER at HiSOR storage ring are presented. The spatial distributions of radiation at 400, 450, 600, 700 and 950 $\mathrm{nm}$ were recorded and analysed. Horizontally and vertically polarized components of ER were compared with numerical results, which were obtained by the new complex of computer codes SMELRAD (SiMulation of ELectromagnetic RADiation). These codes employ the same approach as the codes for wiggler radiation [29]. They include both accelerator and velocity terms of radiation field and allow an accurate and fast simulation of vast variety of edge and wiggler radiation characteristics in real (experimentally measured) magnetic fields. An electron beam emittance and nearfield effects may also be taken into account.

\footnotetext{
*Corresponding author. Email: nick@sci.hiroshima-u.ac.jp
}

\section{EXPERIMENTAL SETUP}

The HiSOR electron storage ring [30] is of a racetrack type consisting of two $180^{\circ}$ bending magnets and four quadrupole doublets, see Table 1 and Fig. 1.

\begin{tabular}{ll} 
Table 1: Main parameters of HiSOR storage ring \\
\hline Energy & $700 \mathrm{MeV}$ \\
Circumference & $21.946 \mathrm{~m}$ \\
Bending radius & $0.87 \mathrm{~m}$ \\
Stored current (Max) & $100 \mathrm{~mA}$ \\
Bending field & $2.7 \mathrm{~T}$ \\
Critical wavelength & $1.42 \mathrm{~nm}$ \\
Length of straight section & $8.24 \mathrm{~m}$ \\
\hline
\end{tabular}

ER was extracted at the helical undulator port through the fused-silica window. A plane mirror reflected ER towards CCD camera with $1536 \times 1024$ pixels detector, pixel size is $9 \mathrm{x} 9 \mu \mathrm{m}$ (Fig. 1). A narrow band interference filters with FWHM about $10 \mathrm{~nm}$ were used. ER images were focused by a lens with focal length of $306.3 \mathrm{~mm}$ and $295 \mathrm{~mm}$ for radiation wavelengths $950 \mathrm{~nm}$ and $400 \mathrm{~nm}$ respectively. The total length between the edge of the downstream bending magnet and CCD detector was 3242 $\mathrm{mm}$. Since the positions of the optical elements remained intact in the course of experiments, the changing of bandpass filters varied slightly the location of the ER image plane. A distance $D$ from the edge of magnet to the ER image plane was $1916 \mathrm{~mm}$ and $1997 \mathrm{~mm}$ for the radiation wavelengths $950 \mathrm{~nm}$ and $400 \mathrm{~nm}$ respectively.

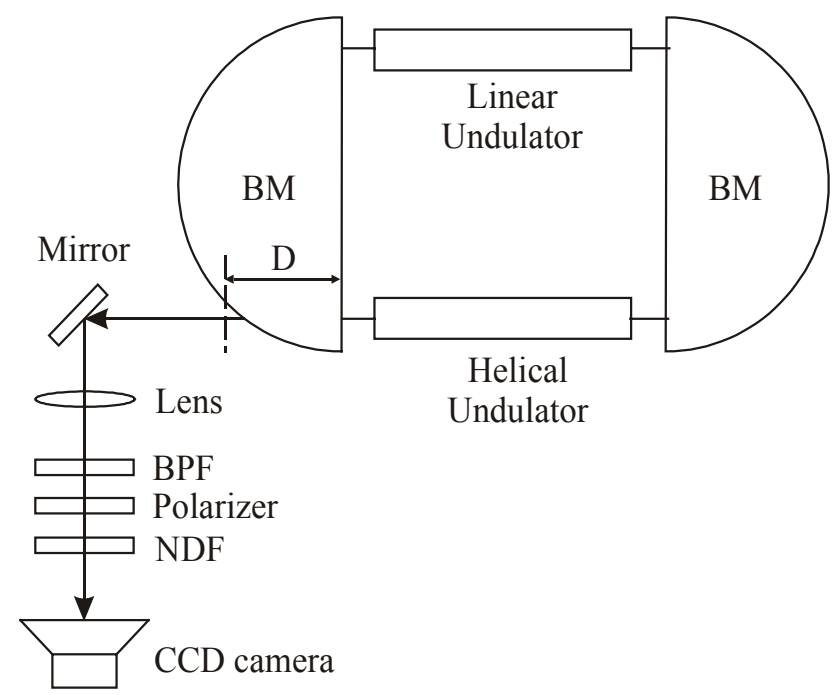

Fig. 1. Layout of ER measurements at HiSOR: BM bending magnets, BPF - band-pass filter, NDF - neutral density filter, dashed line shows the image plane of ER. 


\section{EXPERIMENTAL RESULTS}

Transversal images of ER measured at wavelengths 400 $\mathrm{nm}$ and $950 \mathrm{~nm}$ are shown in Fig. 2 and Fig. 3 respectively. Since the distance $D$ to the image plane is shorter than the straight section length, ER from the downstream bending magnet far exceeds the ER from the upstream magnet.

Numerical simulation of ER at HiSOR shows that the oscillations in intensity due to interference effect should be smoothed by the electron beam emittance. As a result, the distributions of $\sigma$-polarized ER should have one peak at the storage ring median plane, and $\pi$-polarized ER should exhibit two peaks located symmetrically above and below median plane [24]. The measured images of ER spatial distributions (Fig. 2 and Fig. 3) clearly confirm such behaviour. Right side of the pictures corresponds to radiation from the uniform part of the bending field. More intricate shape of ER at $950 \mathrm{~nm}$ wavelength may be attributable to the contribution of radiation from steering magnets and quadrupole magnets, which clearly is of greater intensity than that at $400 \mathrm{~nm}$, although this problem needs further consideration.
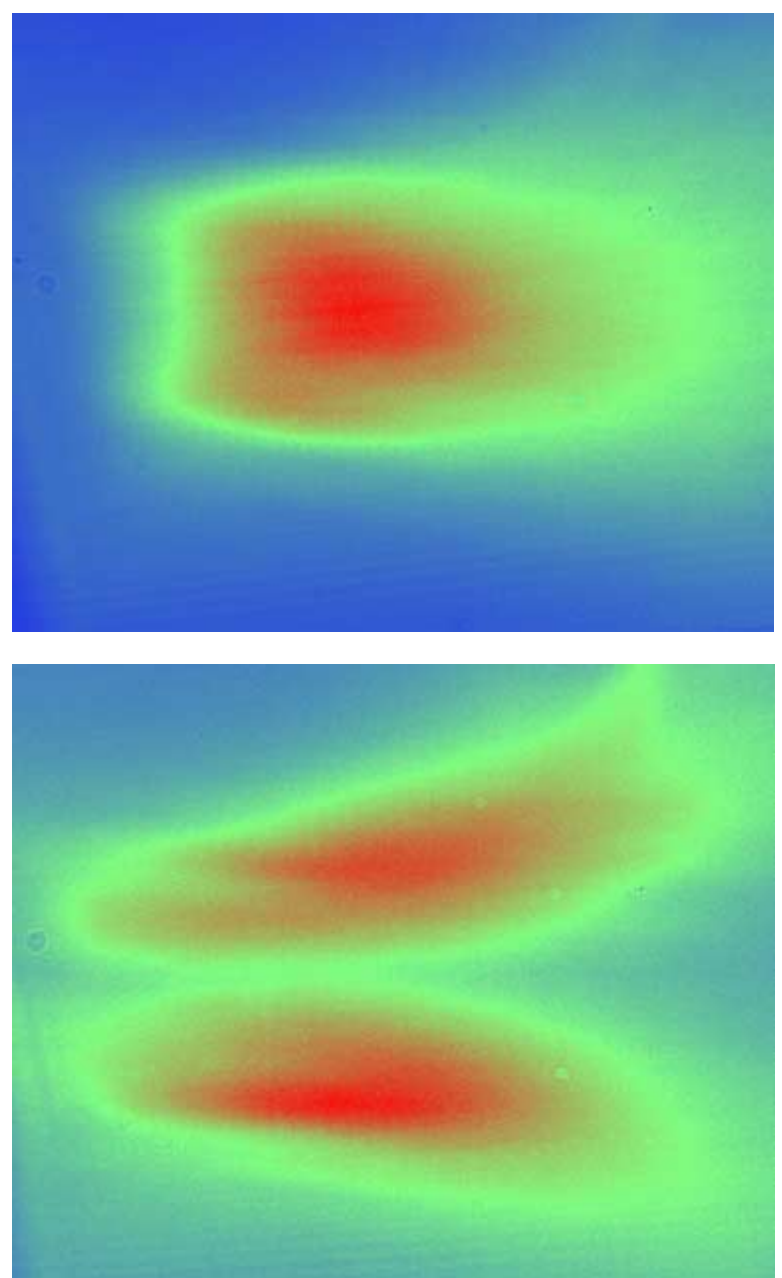

Fig. 2 Measured spatial distribution of $400 \mathrm{~nm}$ ER: $\sigma$-polarized (above) and $\pi$-polarized (below).
Fig. 4 shows the cross-section of $\sigma$-polarized ER along the horizontal line (i.e. in the storage ting median plane). A dashed curve shows numerically simulated distribution. The following electron beam parameters were used for simulation: horizontal and vertical sizes are $1.51 \mathrm{~mm}$ and $0.13 \mathrm{~mm}$ respectively; horizontal and vertical angular spreads are $0.41 \mathrm{mrad}$ and $0.063 \mathrm{mrad}$ respectively. Fig. 5 and Fig. 6 show the vertical cross-sections of $\sigma$-polarized and $\pi$-polarized ER distributions. The experimental and simulation data are in a rather good qualitative agreement, although further analysis is necessary to get a better quantitative coincidence. The vertical distributions (Fig. 5 and Fig. 6) exhibit some asymmetry. It seems that there is an additional source of radiation (probably the steering magnet), which generates additional portion of light a little bit below the median plane. The positive direction of the horizontal axis in Fig. 4 corresponds to region, which is illuminated by the light from uniform magnetic field that is standard synchrotron radiation. It is easy to see that in our case the intensity of $\sigma$-polarized ER with $400 \mathrm{~nm}$ wavelength about twice as large as the correspondent intensity of standard synchrotron radiation.
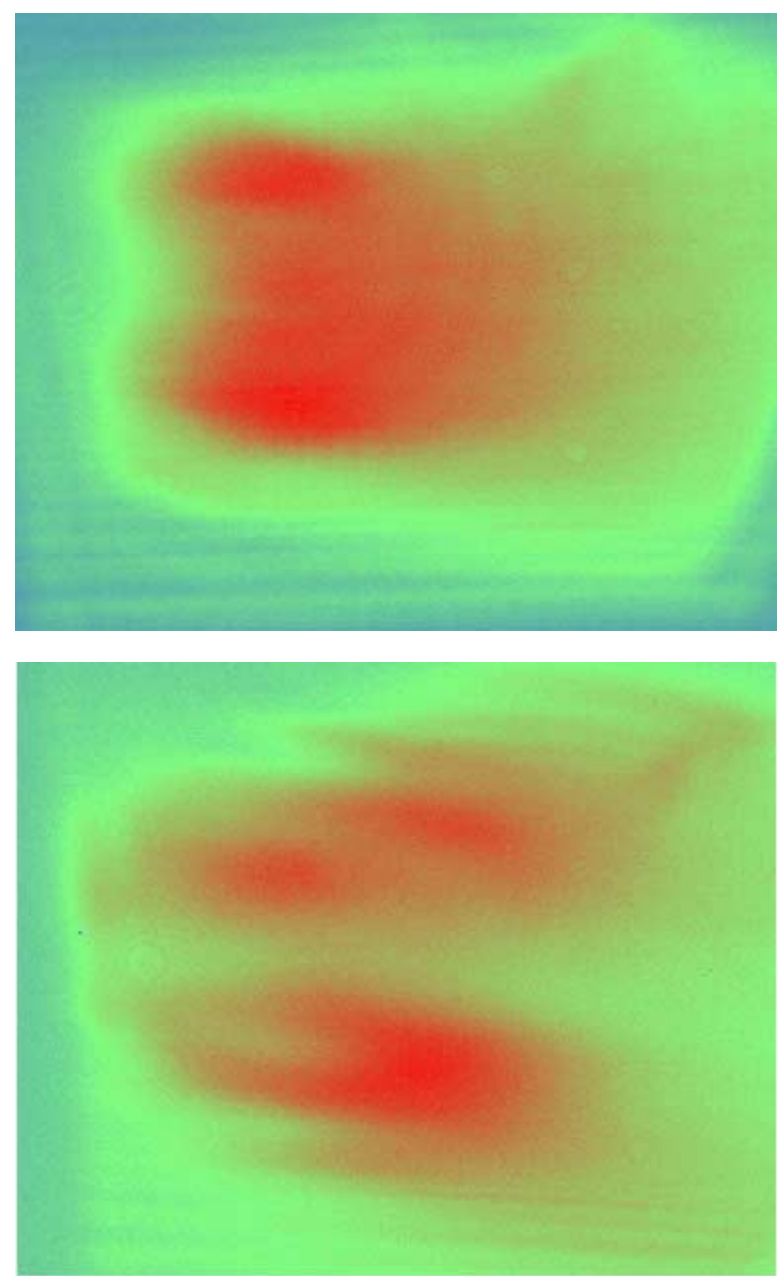

Fig. 3 Measured spatial distribution of $950 \mathrm{~nm}$ ER: $\sigma$-polarized (above) and $\pi$-polarized (below). 


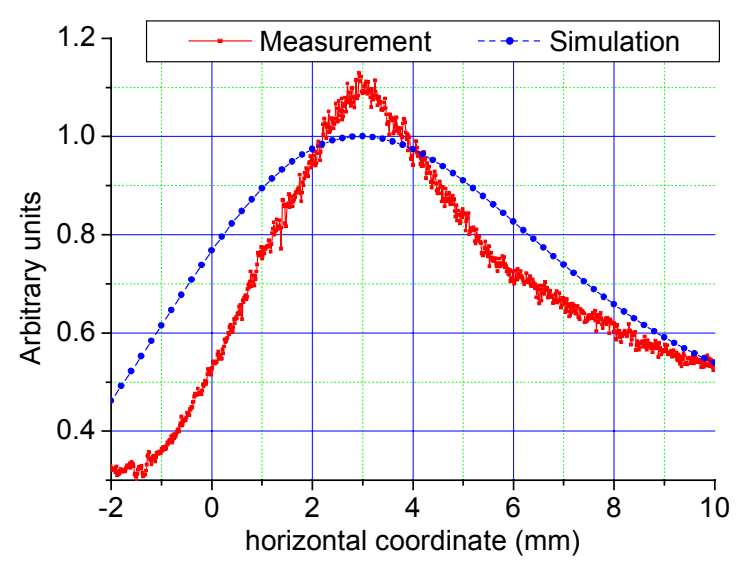

Fig. 4. Intensity distribution of $\sigma$-polarized ER along horizontal line.

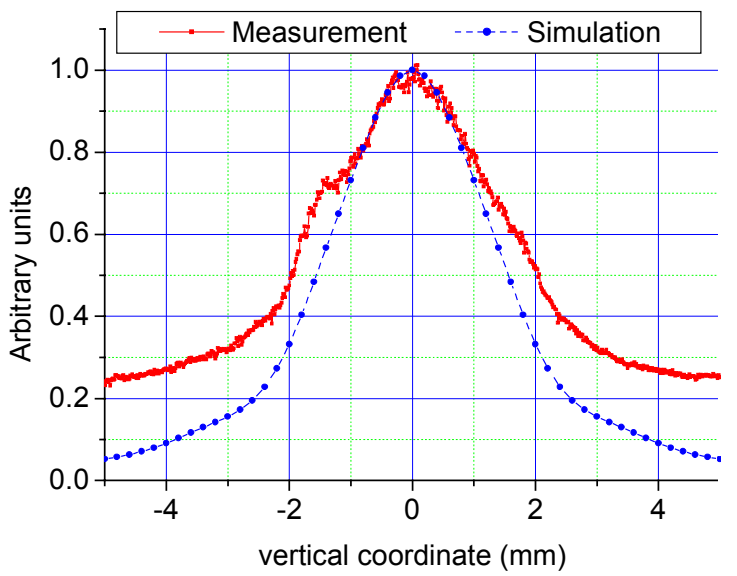

Fig. 5. Intensity distribution of $\sigma$-polarized ER along vertical line.

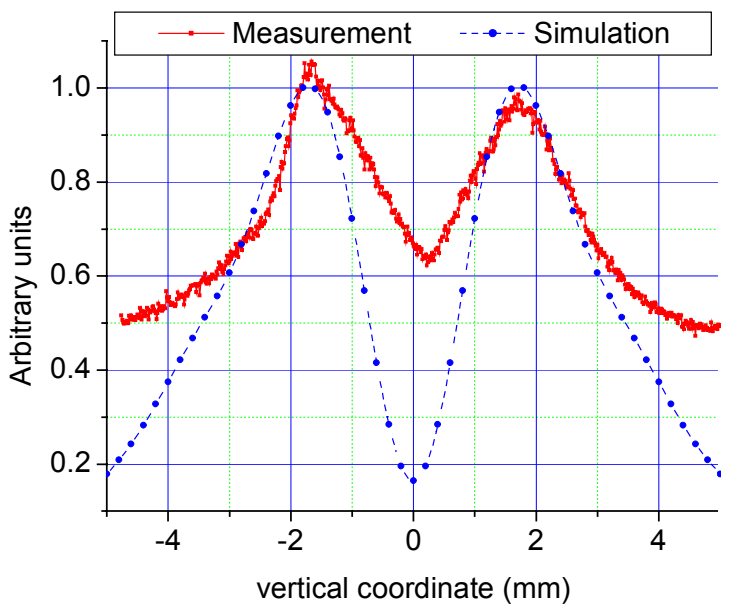

Fig. 6. Intensity distribution of $\pi$-polarized ER along vertical line.

\section{REFERENCES}

[1] R. Bossart et al., Nucl. Instr. \& Meth. 164, 375 (1979).

[2] R. Bossart et al., Nucl. Instr. \& Meth. 184, 349 (1981).

[3] M. M. Nikitin et al., Sov. Tekh. Phys. Lett., 5, 347 (1979).

[4] M. M. Nikitin et al., Sov. Phys. JETP, 52, 388 (1980).

[5] M. M. Nikitin et al., IEEE Trans. Nucl. Sci., NS-28, 3130 (1981).

[6] R. Coisson, Optic Commun., 22, No.2, 135 (1977).

[7] R. Coisson, Phys. Rev., A20, 524 (1979).

[8] N. V. Smolyakov, Sov. Phys. Tekh. Phys., 30, 291 (1985).

[9] N. V. Smolyakov, Sov. Phys. Tekh. Phys., 31, 741 (1986).

[10] N. V. Smolyakov, Sov. Phys. Tekh. Phys., 33, 1320 (1988).

[11] E. G. Bessonov, Sov. Phys. JETP, 53(3), 433 (1981).

[12] V. G. Bagrov et al., Nucl. Instr. \& Meth. 195, 569 (1982)

[13] Yu. A. Bashmakov, Sov. Phys. Tekh. Phys., 31, 310 (1986).

[14] Yu. A. Bashmakov, Rev. Sci. Instr., 63, 343 (1992).

[15] O. V. Chubar and N.V. Smolyakov, J. Optics (Paris), 24, 117 (1993).

[16] O. V. Chubar and N.V. Smolyakov, PAC93 1626.

[17] O. V. Chubar, Rev. Sci. Instr., 66(2), 1872 (1995).

[18] R. A. Bosch and O. V. Chubar, AIP Conf. Proc., 417, 35 (1997).

[19] R.A. Bosch, Nucl. Instr. \& Meth. A386, 525 (1997).

[20] R.A. Bosch, Il Nuovo Cim., D20, 483 (1998).

[21] R.A. Bosch, Nucl. Instr. \& Meth. A431, 320 (1999).

[22] F. Meot, Part. Acceler. 62, 215 (1999).

[23] R.A. Bosch, Nucl. Instr. \& Meth. A454, 497 (2000).

[24] N.V. Smolyakov et al., Nucl. Instr. \& Meth. A448, 73 (2000).

[25] G. Muelhaupt and C. Denise, Nucl. Instr. \& Meth. A387, 319 (1997).

[26] Y.-L. Mathis et al., Phys. Rev. Lett., 80(6), 1220 (1998).

[27] U. Schade et al., Nucl. Instr. \& Meth. A455, 476 (2000).

[28] P. Roy et al., Phys. Rev. Lett., 84(3), 483 (2000).

[29] N. V. Smolyakov, Nucl. Instr. \& Meth. A467-8, 223 (2001).

[30] M. Taniguchi and J. Ghijsen, J. Synchrotron Rad. 5, 1176 (1998). 